\title{
Solving Atomic Broadcast with Indirect Consensus
}

\author{
Richard Ekwall \\ André Schiper \\ nilsrichard.ekwall@epfl.ch \\ andre.schiper@epfl.ch \\ École Polytechnique Fédérale de Lausanne (EPFL) \\ 1015 Lausanne, Switzerland \\ Technical Report LSR/2006/01
}

\begin{abstract}
In previous work, it has been shown how to solve atomic broadcast by reduction to consensus on messages. While this solution is theoretically correct, it has its limitations in practice, since executing consensus on large messages can quickly saturate the system. The problem can be addressed by executing consensus on message identifiers instead of the full messages, in order to decouple the size of the messages from the size of the data sent by the consensus algorithm.

In this paper, we study the impact of executing consensus on message identifiers instead of on the full messages, in the context of solving atomic broadcast. We also discuss the implications of executing consensus on message identifiers on the consensus and atomic broadcast algorithms.
\end{abstract}

\section{Introduction}

\subsection{Context}

Atomic broadcast (or total order broadcast) and consensus are important abstractions in fault tolerant distributed computing. Atomic broadcast ensures that messages that are sent are delivered in the same order by all processes [5]. Consensus allows a group of processes to reach a common decision. In [2], the authors present a reduction of atomic broadcast to consensus. In this reduction, the atomic broadcast algorithm performs consensus runs on sets of messages in order to determine the delivery order of those messages.

${ }^{*}$ A shorter version of this paper appears in Proceedings of the IEEE 2006 International Conference on Dependable Systems and Networks (DSN-2006) 
While this is correct from a theoretical point of view, it is inefficient in practice. Indeed, executing consensus on messages can lead to heavy network usage if the messages are large. Instead, if consensus is executed on message identifiers (indirect consensus), the messages themselves only need to be diffused once and the ordering process is then done on light-weight message identifiers.

Executing consensus on message identifiers has already been done in previous group communication stack implementations [3,9] and has always been seen as being easy, given a consensus algorithm on messages. However, in these group communication implementations, the consensus algorithms were not adapted to handle message identifiers instead of messages. As a consequence, if at least one process can crash, it can lead to a faulty execution, as we show in this paper.

To correctly implement a group communication stack, the consensus and atomic broadcast algorithms need to be adapted to the case where the decision is taken on identifiers instead of messages. We show that these modifications are not trivial for all consensus algorithms and can affect their resilience.

\subsection{Contributions}

We start by discussing and illustrating the advantages of executing consensus indirectly on message identifiers rather than on messages. Two contributions are then presented in this paper: we (1) start by presenting indirect consensus, and show what guarantees it must provide to ensure the correctness of the atomic broadcast algorithm. We then (2) show that the transformation of failure-detector based consensus algorithms on messages into indirect consensus algorithms on message identifiers is far from trivial: the resilience (i.e. the number of supported failures) of some consensus algorithms can be affected by the modifications. To illustrate this, two $\diamond \mathcal{S}$-based consensus algorithms are adapted into indirect consensus algorithms that work on message identifiers. The resilience of one of the algorithms is affected by the modifications whereas the other one isn't.

The paper is structured as follows. In Section 2, we motivate the use of consensus on message identifiers rather than on messages and present the formal specification of indirect consensus. Section 3 illustrates the modifications that are needed to transform two consensus algorithms with the failure detector $\diamond \mathcal{S}$ into indirect consensus algorithms. The section emphasizes the fact that not all consensus algorithms on messages can be trivially modified into indirect consensus algorithms on message identifiers. Section 4 compares the performance of the consensus and indirect consensus algorithms presented in Section 3. Finally, Section 5 concludes this paper. 


\section{Motivation and indirect consensus}

\subsection{Atomic broadcast on message identifiers}

In the following paragraphs, we discuss the use of message identifiers in atomic broadcast algorithms. We start by giving the specifications of reliable and atomic broadcast. We then recall the reduction of atomic broadcast to consensus and show performance comparisons between executions of atomic broadcast using messages and executions using message identifiers. The specifications and the short reminder on the reduction of atomic broadcast to consensus help in understanding the problems involved when executing consensus on message identifiers.

We consider an asynchronous system composed of $n$ processes taken from a set $\Pi=\left\{p_{1}, \ldots, p_{n}\right\}$. The processes communicate by passing messages over reliable channels and can only fail by crashing (no Byzantine failures). A process that never crashes is said to be correct, otherwise it is faulty.

Informally, reliable broadcast guarantees that all correct processes deliver the same set of messages. Formally, reliable broadcast is defined by two primitives rbroadcast and rdeliver and satisfies three properties [5]: (1) Validity: If a correct process $p$ rbroadcasts a message $m$, then it eventually rdelivers $m$, (2) Uniform integrity: For any message $m$, every process $p$ rdelivers $m$ at most once and only if $m$ was previously rbroadcast, (3) Agreement: If a correct process rdelivers $m$, then all correct processes eventually rdeliver $m$.

(Uniform) atomic broadcast is reliable broadcast augmented with a uniform agreement property and a total order property. The (uniform) atomic broadcast problem is defined by two primitives abroadcast and adeliver that satisfy the (1) Validity and (2) Uniform integrity properties of reliable broadcast and the additional uniform agreement and order properties: (3) Uniform Agreement: If a process (correct or not) adelivers $m$, then all correct processes eventually adeliver $m$, and (4) Uniform Total Order: If some process, correct or faulty, adelivers $m$ before $m^{\prime}$, then every process adelivers $m^{\prime}$ only after it has adelivered $m$.

In [2], the authors present a reduction of atomic broadcast to consensus. In this reduction, whenever a message $m$ is abroadcast, $m$ is reliably broadcast to all processes. Following this, whenever a process receives a message that it hasn't already adelivered, it executes consensus in order to reach a decision with the other processes on the next message to adeliver. In the reduction of atomic broadcast to consensus in [2], the processes thus execute a sequence of consensus runs on sets of messages as long as new messages are abroadcast.

This reduction is correct. However, in a system where the messages are large, the consensus runs are executed on sets of large messages. Thus, the size of the data exchanged by the consensus algorithm is large and can potentially saturate the 


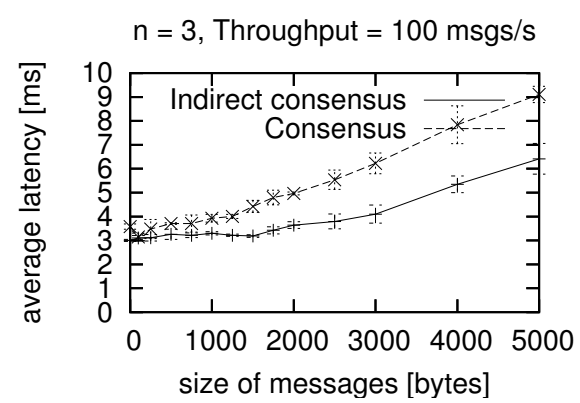

(a) $100 \mathrm{msgs} / \mathrm{s}$

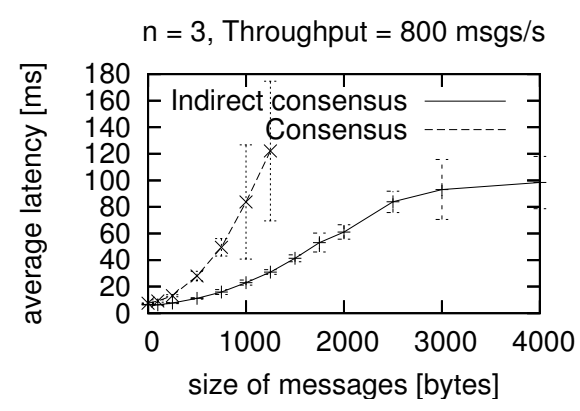

(b) $800 \mathrm{msgs} / \mathrm{s}$

Figure 1: Latency of the atomic broadcast algorithm versus size of the messages in a system with 3 processes

system. In order to avoid this, consensus can be executed on message identifiers instead of the messages themselves. This decouples the size of the messages from the size of the data exchanged by consensus. Since the relationship between the messages and their identifiers is bijective, the delivery order of the messages can easily be inferred from the ordered sequence of message identifiers (which ensures the Uniform Total Order property of atomic broadcast).

The performance gain when using message identifiers instead of messages in consensus is not negligible. Indeed, the size of a message identifier is independent of the size of the message itself. Thus, the size of the data exchanged by consensus remains constant as the size of the messages increases. Figure 1 illustrates the performance difference between executing consensus on messages or message identifiers in the context of atomic broadcast. The performance metric for atomic broadcast is the latency, defined as the average (over all processes) of the elapsed time between abroadcasting a message $m$ and adelivering $m$. The figure shows the latency of atomic broadcast as a function of the size of the messages. The results are shown for two throughputs (the overall rate of atomic broadcasts in the system: 100 or 800 messages per second). The tests were done using the Neko framework on a local area network with Pentium III machines. More details on the framework and the system setup can be found in Section 4.

One can clearly see that as the size of the messages increases, the latency of consensus on message identifiers is lower than the latency when using entire messages. This result becomes clearer as the throughput of atomic broadcasts increases and as the size of the system increases. As a consequence, except for trivial conditions (low throughputs and small systems), executing consensus on message identifiers rather than on entire messages is clearly justified. 


\subsection{Violating validity of atomic broadcast}

Executing consensus on message identifiers implies that the consensus and atomic broadcast algorithms must be adapted to explicitly handle message identifiers instead of messages, as we now show. More specifically, we show that if the atomic broadcast algorithm directly executes the original consensus algorithm in [2] on message identifiers, the validity property of atomic broadast could be violated. Imagine that a faulty process $p$ reliably broadcasts a new message $m$ and starts executing consensus on its message identifier $i d(m)$. Let us assume that the consensus algorithm decides on $i d(m), p$ crashes and no other process receives a copy of $m$. No other process than $p$ is able to deliver $m$ (and thus any message ordered after $m$ ). Furthermore, in order to guarantee the total order property of atomic broadcast, $i d(m)$ cannot be removed from the sequence of ordered message identifiers. As a consequence, the validity property of atomic broadcast is violated in such an execution, since $m$ and any following message in the ordered sequence cannot be delivered.

The problem described above could be avoided by using uniform reliable broadcast instead of reliable broadcast in the atomic broadcast algorithm. Uniform reliable broadcast guarantees that if at least one process (correct or not) delivers a message, then all correct processes eventually deliver that message [5]. Since the atomic broadcast algorithm in [2] only executes consensus on messages that have been uniformly reliably delivered, this solution guarantees that all correct processes eventually receive a copy of any message ordered by consensus. However, the cost of using uniform reliable broadcast is higher than that of reliable broadcast (this is later illustrated in Section 4.4).

Instead of using uniform reliable broadcast and incurring its cost, we suggest to adapt the consensus algorithm to handle message identifiers and provide additional properties that ensure the correctness of atomic broadcast.

\subsection{Indirect consensus}

The motivation of introducing indirect consensus is to capture the differences between executing consensus on messages and on message identifiers. Instead of executing consensus directly on messages, we want to indirectly execute consensus on message identifiers. Simultaneously, indirect consensus has to offer guarantees to atomic broadcast so that all the messages whose identifiers have been ordered can be delivered by atomic broadcast.

In indirect consensus, each proposal is a pair $(v, r c v)$, where $v$ is a set of message identifiers (and $m s g s(v)$ are the messages whose identifiers are in $v$ ). $r c v$ is a function such that $r c v(v)$ returns true only if the process has received $m s g s(v)$. Whenever a decision is taken on $v$, indirect consensus must ensure that all correct 
processes eventually receive $m s g s(v)$. In the context of indirect consensus, we introduce the following hypothesis on the $r c v$ function:

Hypothesis A: If $r c v(v)$ is true for a correct process, then $r c v(v)$ is eventually true for all correct processes.

Formally, we specify indirect consensus similarly to consensus, in terms of two primitives: propose(v,rcv) and decide(v). The (uniform) indirect consensus problem is then specified by five properties. The four first properties are (almost) identical to (uniform) consensus:

Termination : If the Hypothesis A holds, then every correct process eventually decides some value.

Uniform integrity : Every process decides at most once.

Uniform agreement : No two processes (correct or not) decide a different value.

Uniform validity: If a process decides $v$, then $(v, r c v)$ was proposed by some process in $\Pi$.

No loss : If a process decides $v$ at time $t$, then one correct process has received $m s g s(v)$ at time $t$.

The No loss property implies that indirect consensus has to be able to know if given $v$, the messages $m s g s(v)$ have been received. This information is provided by the $r c v$ function (the function would typically be provided by the atomic broadcast algorithm).

\subsection{Reducing atomic broadcast to indirect consensus}

The reduction of atomic broadcast to indirect consensus is almost identical to the reduction of atomic broadcast to consensus in [2]. The main difference resides in the fact that instead of executing consensus on a set of messages, indirect consensus is executed on a pair (set of message identifiers, rcv function). The validity of atomic broadcast is ensured by the No loss property of indirect consensus: the messages in $\operatorname{msgs}(v)$ corresponding to the decision $v$ of indirect consensus are rdelivered by at least one correct process (and thus all correct processes eventually rdeliver msgs $(v)$ ).

The atomic broadcast algorithm using indirect consensus is shown in Algorithm 1. It shows the following: whenever abroadcast is called on a message $m$, then $m$ is rbroadcast to all processes (line 8). If a process rdelivers a message 


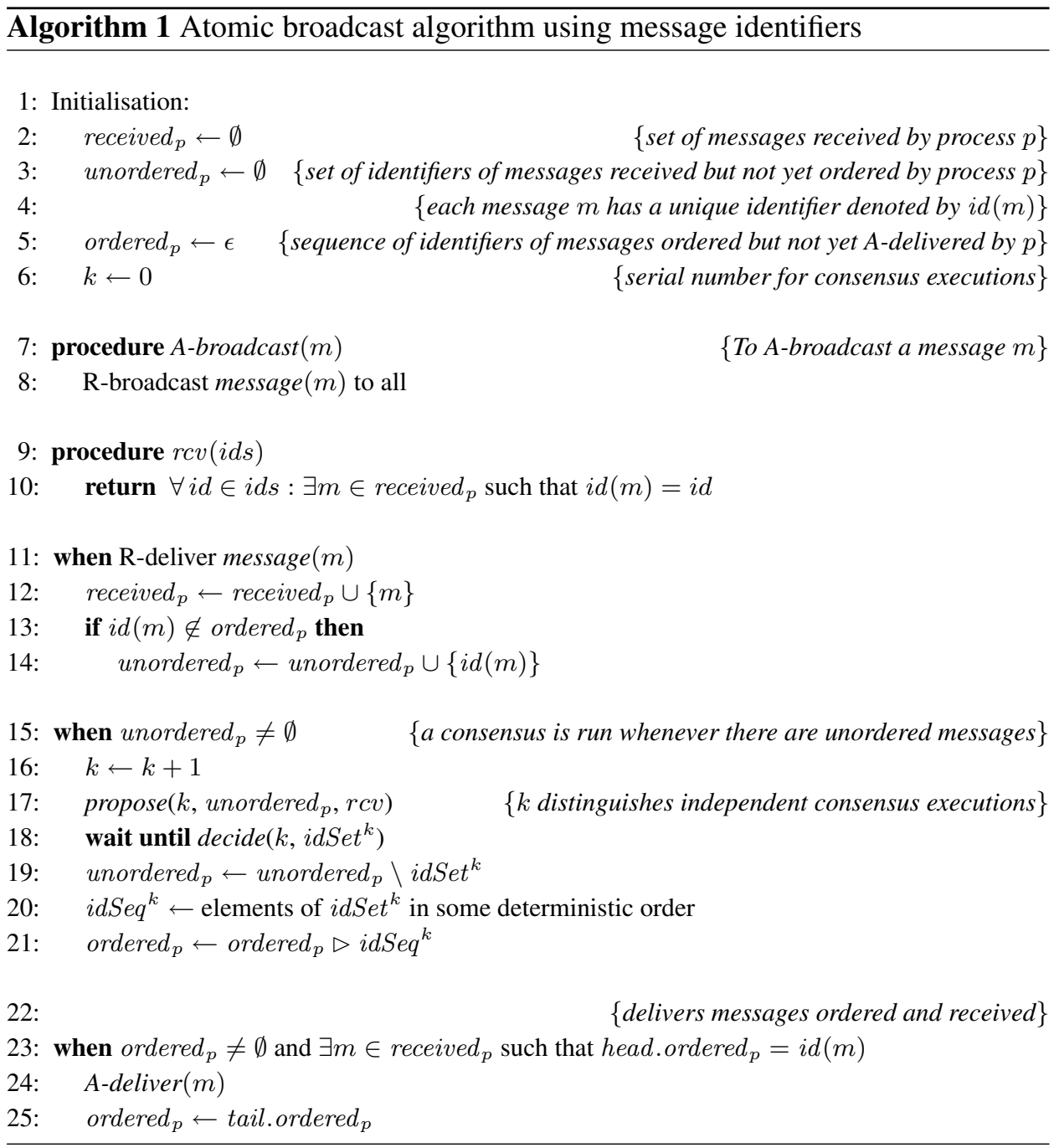

that hasn't been ordered yet (line 13), consensus is executed on the unordered message identifiers (lines 15 to 18). The $r c v$ function is shown in lines 9 and 10 of Algorithm 1.

We now show that the $r c v$ function of atomic broadcast satisfies the Hypothesis $A$ above. If $r c v(v)$ is true for a correct process, then all messages $m s g s(v)$ whose identifier is in $v$ have been previously rdelivered. Following the Agreement property of reliable broadcast, all correct processes eventually rdeliver msgs $(v)$ and thus $r c v(v)$ is eventually true for all correct processes.

\section{Solving indirect consensus}

We now show how to solve indirect consensus. We start by discussing what properties an indirect consensus algorithm must enforce in order to guarantee the No 
loss property. Two consensus algorithms are then adapted into indirect consensus algorithms: (1) the Chandra-Toueg $\diamond \mathcal{S}$ consensus algorithm $(C T)$ and (2) the Mostéfaoui-Raynal $\diamond \mathcal{S}$ consensus algorithm $(M R)$. These two algorithms illustrate two cases. CT illustrates the case of a consensus algorithm that is fairly easy to adapt into an indirect consensus algorithm; MR illustrates the case of a consensus algorithm whose resilience is reduced by the adaptation into an indirect consensus algorithm (the indirect consensus algorithm requires $\left\lceil\frac{2 n+1}{3}\right\rceil$ correct processes where the original consensus algorithm required $\left\lceil\frac{n+1}{2}\right\rceil$ correct processes).

\subsection{Conditions on the correctness of indirect consensus algorithms}

We present the conditions that indirect consensus algorithms must fulfill in order to ensure the No loss property. To do this we introduce the two following definitions:

Definition: $v$-valent configuration. As in [4], we say that a configuration is $v$ valent at time $t$ if any decision that is taken after $t$ can only be $v$. As an example, consider a configuration where all processes start consensus at time $t_{0}$ with the same initial value $v$. Such a configuration is $v$-valent at time $t_{0}$ (although the first process to decide only does so after $t_{0}$ ).

Definition: $v$-stable configuration. We say that a configuration is $v$-stable at time $t$ if $f+1$ processes have received $\operatorname{msgs}(v)$ at time $t$ ( $f$ is the maximum number of processes that may crash). $v$-stability ensures that at least one correct process has received $m s g s(v)$.

From these definitions, if a configuration is $v$-valent or $v$-stable at time $t$, any configuration at time $t^{\prime}>t$ is also $v$-valent, respectively $v$-stable.

Ensuring the No loss property We now show that for the No loss property to hold, it is necessary and sufficient that any configuration that is $v$-valent at some time $t$ is also $v$-stable at $t$.

We first show that the No loss property of an algorithm holds, if the algorithm guarantees that a $v$-valent configuration is also $v$-stable. Let us assume that the first decision on a value $v$ is taken at some time $t_{0}$. From the Uniform agreement property of the indirect consensus algorithm, the configuration is $v$-valent at time $t_{0}$. Since the $v$-valence of a configuration implies that the configuration is also $v$-stable, $v$-stability also holds at time $t_{0}$. Thus, the No loss property holds.

Now, we show that if an algorithm allows a $v$-valent configuration that is not $v$-stable, then the No loss property of the algorithm does not hold. Let us assume that the system reaches a $v$-valent configuration at time $t$ that is not $v$-stable. Since the configuration is not $v$-stable, at most $f$ processes have received $m s g s(v)$ at 
time $t$. If those $f$ processes crash, all copies of $\operatorname{msgs}(v)$ are lost and no correct process ever receives $m s g s(v)$. Either no decision is taken after time $t$ (and the Termination property of the algorithm is violated) or a decision is taken on $v$, since the system is $v$-valent at time $t$. Since $m s g s(v)$ are never received by a correct process, the No loss property of the algorithm is violated in the latter case.

As a consequence of this result, any indirect consensus algorithm needs to ensure that the relationship " $v$-valence $\Rightarrow v$-stability" for any configuration holds. This relationship between $v$-valence and $v$-stability is not trivially satisfied by a consensus algorithm.

\subsection{Adapting Chandra-Toueg's $\diamond \mathcal{S}$ consensus algorithm}

The following paragraphs present the modification of the CT $\diamond \mathcal{S}$ consensus algorithm [2] into an indirect consensus algorithm. First of all, a brief overview of the original $\diamond \mathcal{S}$ consensus algorithm is presented. Then, the necessary modification to that algorithm and $v$-valence and $v$-stability are discussed. Finally, the adapted indirect consensus algorithm is presented and proved.

\subsubsection{Chandra-Toueg's $\diamond \mathcal{S}$ consensus algorithm}

In [2], the authors present a consensus algorithm based on the unreliable failure detector $\diamond \mathcal{S}$. The algorithm proceeds in rounds and requires a majority $\left(f<\frac{n}{2}\right)$ of correct processes. It behaves as follows: at the beginning of each round, each process sends its estimate of the decision to the process acting as a coordinator in that round. The coordinator waits for a majority of estimates and selects the most recent one (based on its timestamp) and sends it to all processes. At this point, each process either receives the coordinator's proposal, or suspects the coordinator of having crashed. In the former case, the process sets its own estimate to the coordinator's proposal, updates its timestamp and sends a positive acknowledgement (ack) to the coordinator. In the latter case, a negative acknowledgement (nack) is sent. In both cases, the non-coordinator processes proceed to the next round.

The coordinator waits for a majority $(f+1)$ of answers. If all answers are acks, the coordinator decides and informs the other processes of its decision. If at least one nack is received, the coordinator proceeds to the next round without deciding. It is easy to show that if $\left\lceil\frac{n+1}{2}\right\rceil$ of processes have accepted the coordinator's proposal $v$, then the system is in a $v$-valent configuration (i.e. any future decision is $v$ ), although the decision on $v$ might only be taken in a later round. 


\subsubsection{Adapting the algorithm into an indirect consensus algorithm}

In the original CT algorithm, a process that receives the coordinator's proposal in a given round updates its own estimate to match the proposal of the coordinator (and sends an $a c k$ ). This is precisely the operation that allows the incorrect scenario presented in Section 2.2 to occur. Indeed, if all processes blindly adopt the coordinator's proposal $v$ (thus leading to a $v$-valent configuration, with $v$ a set of message identifiers) and that the originator of $m s g s(v)$ crashes, then $m s g s(v)$ might be lost and no $v$-stable configuration of the system can be reached.

In order to avoid this situation, we propose the following modification: whenever a process receives the coordinator's proposal $v$, it checks if $m s g s(v)$ have been received (using the $r c v$ function). If so, an ack is sent to the coordinator (the proposal is accepted); otherwise, a nack is sent (the proposal is refused). Similar approaches have been taken in $[1,6]$.

The modified algorithm The pseudo-code of the adapted indirect consensus algorithm is shown in Algorithm 2 (the parts that were modified with respect to the original CT algorithm have bold line numbers) and is expressed as in [8].

The lines 25 to 30 correspond to the modification described above. The $r c v$ function is called at line 25 to test if all messages whose identifiers are in the coordinator's proposal have been received. The additional variable estimate $_{c}$ (lines 2 , 18, 20, 21 and 37) represents the coordinator's proposal and can be different from the coordinator's own estimate estimate $_{p}$ (in case the coordinator does not have the messages corresponding to the estimate $v$ with the highest timestamp). This is explained in the next paragraph.

The need for estimate $_{c}$ and estimate $_{p} \quad$ Consider a coordinator $c$ at line 21 that sends $v$ to all in round 1 ( $c$ has received $m s g s(v)$ ), and a process $p_{i}$ that accepts this estimate at line $25\left(p_{i}\right.$ has received $\left.m s g s(v)\right)$. In round 2 , the coordinator $c^{\prime}$, if it receives the estimate from $c$ or $p_{i}$ selects it, even if it has not received $m s g s(v)$. However, if $c$ and $p_{i}$ crash later, and no other process has received $m s g s(v)$, no correct process might ever receive msgs $(v)$. So, in round 2 the coordinator $c^{\prime}$ updates estimate $e_{c}$ with $v$, but estimate $_{p}$ is still equal to a different value. Once $c$ and $p_{i}$ crash, the estimate $v$ with timestamp 1 will disappear, and an estimate with timestamp 0 will again be chosen.

This scenario illustrates that a process, including the coordinator, only accepts to modify its estimate if it has all the messages corresponding to the identifiers in the new estimate. Since only non-crashed processes send their estimates to the coordinator at the beginning of each round, eventually, only estimates adopted by at least one correct process are received by the coordinator. 


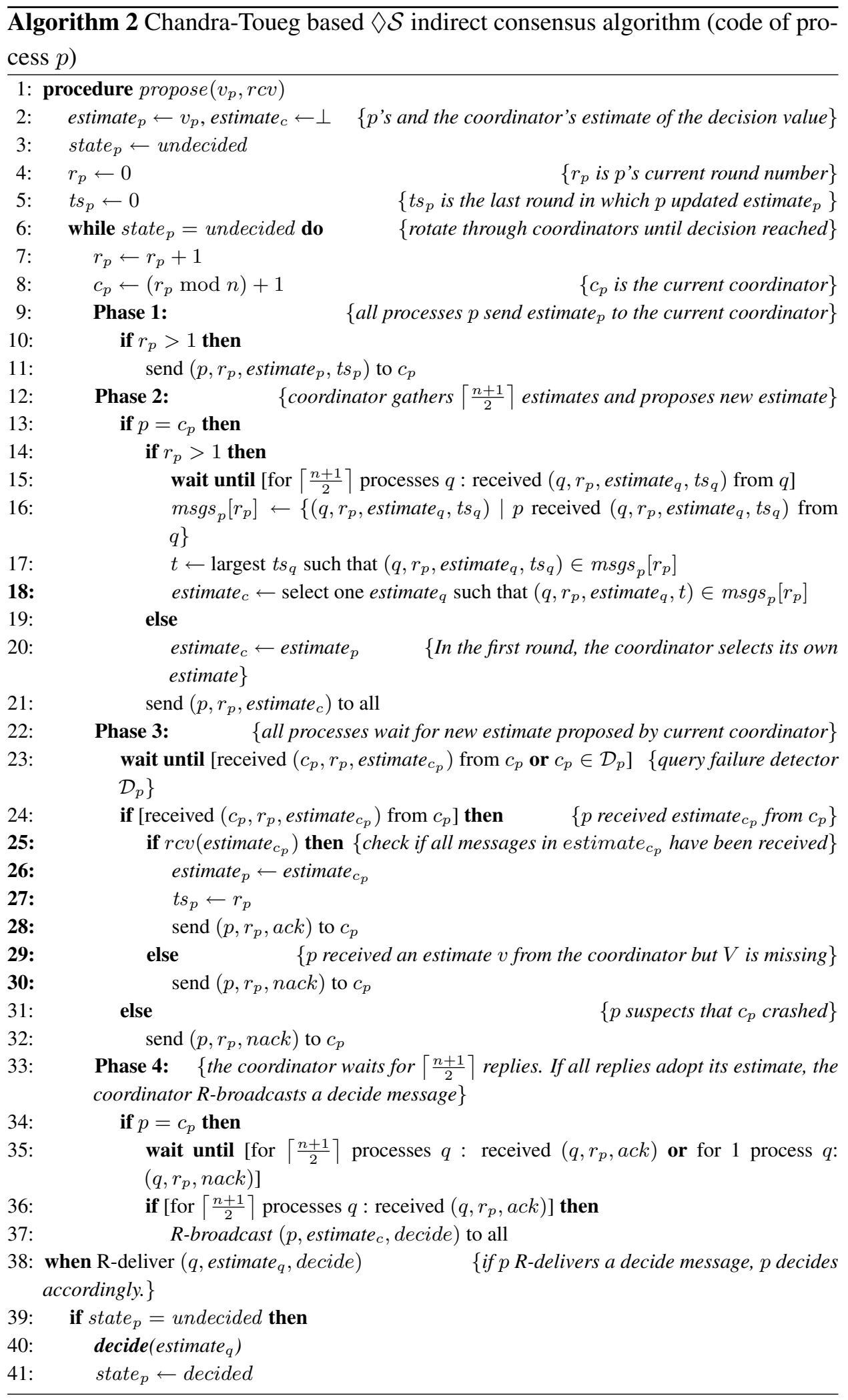




\subsubsection{Proof of the algorithm (sketch)}

The Uniform integrity and Uniform validity properties are trivially proven and are not shown here.

Uniform Agreement The modifications to the algorithm do not affect the uniform agreement properties of the algorithm. Consequently, the proof of the modified algorithm is the same as for the original algorithm by Chandra and Toueg [2].

Indeed, the modified algorithm adds a condition on the acceptance of the coordinator's proposal (and the sending of an $a c k$ ). As a consequence, if a decision is taken in the modified algorithm, it would also have been taken in the original algorithm. Thus, if Uniform agreement holds in the original algorithm, it also holds in the modified algorithm.

Termination There is a time $t$ such that all faulty processes have crashed. After this time $t$, all correct processes have an estimate $v$ such that $r c v(v)$ holds. From Hypothesis $A$, there is thus a time $t^{\prime}$ such that $r c v(v)$ holds for all correct processes and for the estimate $v$ of any correct process. After this time $t^{\prime}$, the indirect consensus algorithm behaves exactly like the original consensus algorithm. Thus, if a decision hasn't been taken before $t^{\prime}$, the Termination property of Chandra-Toueg's $\checkmark \mathcal{S}$ consensus algorithm guarantees that the indirect consensus algorithm terminates.

No loss We show that any $v$-valent configuration is also $v$-stable. If a configuration is $v$-valent, it implies that the coordinator always selects $v$ as its proposal. Since the proposal selected by the coordinator is one of the $\left\lceil\frac{n+1}{2}\right\rceil$ received estimates, and that the coordinator always receives $v$ at least once, at least a majority of processes have an estimate equal to $v$.

These $\left\lceil\frac{n+1}{2}\right\rceil$ processes that have an estimate equal to $v$ can either (1) have started consensus with $v$ or (2) adopted the proposal $v$ of a previous coordinator, in which case the $r c v$ function on $v$ is verified. In both cases, $m s g s(v)$ have been received by a majority of processes and the configuration is $v$-stable. Since any $v$-valent configuration is also $v$-stable, the No loss property holds.

\subsection{Adapting Mostéfaoui-Raynal's $\diamond \mathcal{S}$ consensus algorithm}

We start by presenting an informal overview of the original MR consensus algorithm. The problems encountered when adapting this algorithm into an indirect consensus algorithm are then discussed. The solution to these problems, which modifies the resilience of the algorithm, is then presented. Finally, the adapted indirect consensus algorithm is presented and proven correct. 


\subsubsection{Mostéfaoui-Raynal's $\diamond \mathcal{S}$ consensus algorithm}

In [7], the authors present a consensus algorithm based on unreliable failure detectors and quorums. We consider their $\diamond \mathcal{S}$ based algorithm here. As in the CT consensus algorithm, the MR algorithm proceeds in rounds and requires a majority $\left(f<\frac{n}{2}\right)$ of correct processes. In rounds without failures and without suspicions, a decision can be taken within two communication steps by all processes.

Each round consists of two phases. At the beginning of Phase 1, the coordinator of that round sends its estimate to all processes. Each process then either receives the coordinator's proposal, or suspects the coordinator of having crashed. In the latter case, the process considers that an invalid value $(\perp)$ was received from the coordinator. In both cases, the process sends the estimate received from the coordinator (a valid value or $\perp$ ) to all processes, which concludes Phase 1 of the algorithm.

In Phase 2, each process waits for a majority of estimates (including the one possibly received from the coordinator). If all received estimates are the same value $v$, the process decides $v$ and informs all other processes of its decision. If this is not the case, but at least one received estimate is valid (not $\perp$ ), the process sets its own estimate to the received valid estimate and proceeds to the next round.

The Uniform agreement property of consensus is ensured by the fact that if a decision on $v$ is taken by a process $p$, then $p$ has received the estimate $v$ from $\left\lceil\frac{n+1}{2}\right\rceil$ processes. This in turn ensures that all processes have received at least one estimate equal to $v$ and have thus set their own estimate to $v$. Since the estimates of all processes are equal to $v$, any subsequent decision can only be done on $v$.

\subsubsection{Problems adapting the Mostéfaoui-Raynal consensus algorithm into an indirect consensus algorithm}

As described above, one of the constraints for Uniform agreement to hold in the MR consensus algorithm is that any process that receives at least one valid estimate must accept that estimate, i.e. modify its own estimate to match the received one. Accepting such an estimate might however lead to a violation of the No loss property of indirect consensus. This is shown by two executions that are indistinguishable for some process $p$ : in one execution the configuration is $v$-valent but not $v$-stable; in the other execution the configuration is $v$-valent and $v$-stable.

We assume a system with $n$ processes and $p$ a non-coordinator process in the current round of the algorithm. Process $p$ suspects the coordinator and $p$ does not have the messages corresponding to the coordinator's proposal $v$. The two executions are the following:

(1) the coordinator is correct. $\left\lceil\frac{n+1}{2}\right\rceil$ processes accept the proposal of the coordinator, whereas $\left\lfloor\frac{n-1}{2}\right\rfloor$ suspect the coordinator. The coordinator receives $\left\lceil\frac{n+1}{2}\right\rceil$ 
estimates equal to its own proposal whereas $p$ receives one estimate equal to the coordinator's proposal and $\left\lfloor\frac{n-1}{2}\right\rfloor$ invalid $\perp$ values. In this execution, the coordinator decides. To guarantee the Uniform agreement property of consensus, $p$ must accept the coordinator's proposal $v$ (and thus modify its own estimate), although it doesn't have $m s g s(v)$.

(2) the coordinator is faulty. Let us assume that the $n-1$ non-coordinator processes suspect the coordinator and do not have the messages corresponding to the coordinator's proposal $v$. They thus all send a $\perp$ value. Process $p$ receives the coordinator's proposal as well as $\left\lfloor\frac{n-1}{2}\right\rfloor$ invalid $\perp$ values. If the coordinator crashes before any process receives msgs $(v)$, then $m s g s(v)$ might be lost. In this execution, $p$ must not accept the coordinator's proposal $v$.

If $p$ takes a conservative approach and only accepts a proposal $v$ if it has $m s g s(v)$ (or that at least one correct process has $m s g s(v)$ ), then the Uniform agreement property would be violated in the first execution. If, on the other hand, $p$ takes the optimistic approach of accepting a proposal $v$ even if it doesn't have $m s g s(v)$, this could lead to a $v$-valent configuration that is not $v$-stable. The No loss property of indirect consensus could thus be violated. Therefore, any of the approaches that the algorithm chooses to implement leads to the violation of one of the indirect consensus properties.

The modifications must thus ensure both of the following properties: (i) a process should only accept $v$ if it has $m s g s(v)$ or $f+1$ processes have $m s g s(v)$; (ii) if a process decides $v$ in round $r$, then all non-crashed processes must adopt $v$ during round $r$. Property (i) and (ii) aim at guaranteeing No loss, respectively Uniform agreement.

\subsubsection{Modified Mostéfaoui-Raynal algorithm}

Consequences on the resilience Thus, we must modify the MR algorithm. In Phase 1 of the algorithm, a process can only accept the coordinator's proposal $v$ if it has received $m s g s(v)$ (at this point, a process does not know if any other process has adopted $v$ and can therefore not know if $v$ is stable, which is the second possible condition for adopting $v$ ). Thus, at the end of Phase 1 , when a process $p$ sends the estimate $v$ to all processes, this estimate is a non- $\perp$ value only if $p$ has received $\operatorname{msgs}(v)$.

In Phase 2 of the consensus algorithm, all processes wait for $n-f$ estimates from the other processes. If all of these estimates are identical, a decision can be taken. If they are not, a process $p$ can accept a valid estimate $v$ if (1) $p$ has received $m s g s(v)$ or (2) if the estimate $v$ was received from at least $f+1$ processes (i.e. from at least one correct process that has received $m s g s(v)$ ).

To ensure Uniform agreement, we have seen that if a decision is taken on $v$, 


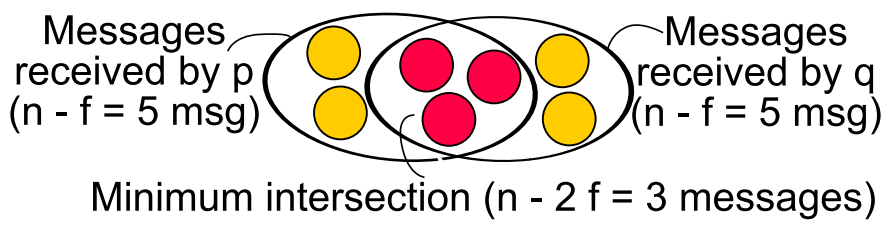

Figure 2: Intersection of the estimates received by two processes $p$ and $q,(n=7$ processes and $f=2$ above). The size of the received estimates set of each process is five $(n-f)$ and the size of the intersection is at least equal to three $(n-2 f)$.

then all processes must accept $v$ as their own estimate. Not all processes have necessarily received $m s g s(v)$ (which means that condition (1) above might not be true for all processes). Therefore, if a decision is taken, the algorithm must ensure that the condition (2) above is true for all processes (i.e. all processes receive at least $f+1$ estimates equal to $v$ ). This can be ensured as follows.

Each process waits for $n-f$ estimates at the beginning of Phase 2. Since there are at most $n$ estimates in the system, each pair of processes receives a common set of estimates. The minimum size of this common set is $n-2 f$ (assuming $f<\frac{n}{2}$ ), as illustrated in Figure 2 (in the case of a system with $n=7$ processes and at most $f=2$ failures). So condition (2) is ensured if $n-2 f \geq f+1$, which leads to $f<\frac{n}{3}$.

The modified MR algorithm The pseudo-code of the adapted MR indirect consensus algorithm is shown in Algorithm 3 (the parts that were modified with respect to the original algorithm have bold line numbers) and is expressed as in [8].

The lines 16 to 19 correspond to the modifications to the first phase of the algorithm. With these modifications, a process accepts the coordinator's proposal $v$ only if it received $m s g s(v)$ (in the original consensus algorithm, $v$ was always accepted). In Phase 2, the modifications are two-fold. First of all, the condition $f<\frac{n}{3}$ force each process to wait for $\left\lceil\frac{2 n+1}{3}\right\rceil$ estimates at the beginning of Phase 2 (lines 21 and 22). Secondly, if a process receives a valid estimate $v$ as well as $\perp$ values, the valid estimate is adopted if (1) the process has $m s g s(v)$ or (2) if the estimate $v$ was received from more than one third of the processes (lines 28 and 29).

The remaining parts of the indirect consensus algorithm are identical to the original MR consensus algorithm.

\subsubsection{Proof of the algorithm (sketch)}

The Uniform integrity and Uniform validity properties are trivially proven and are therefore not shown here. 


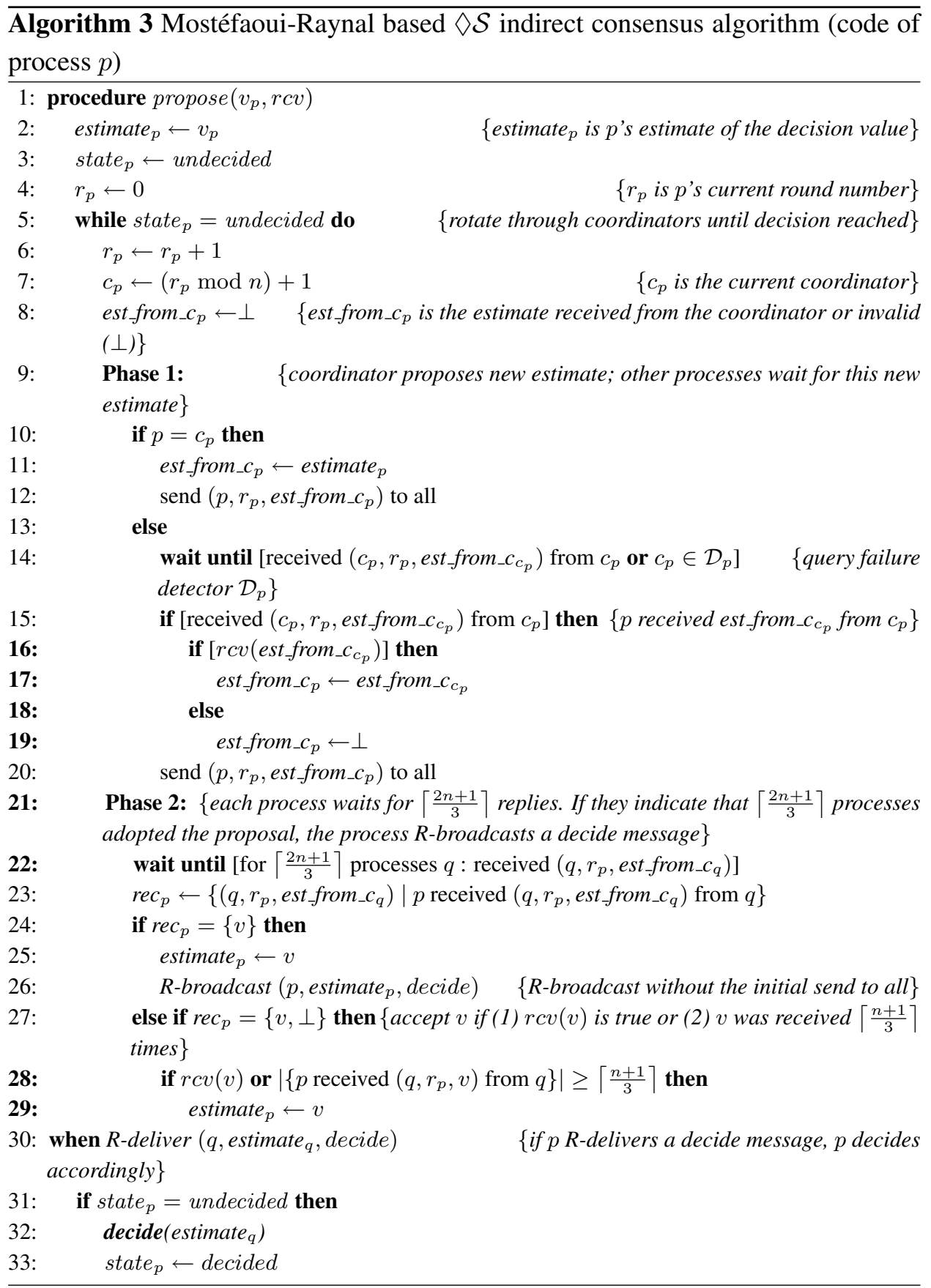

Uniform agreement Let process $p$ be the first process that reliably broadcasts a decision message and then decides on some value $v$. Process $p$ previously received the estimate $v$ from $\left\lceil\frac{2 n+1}{3}\right\rceil$ processes in Phase 2 of a given round $r$. All other processes also received $\left\lceil\frac{2 n+1}{3}\right\rceil$ values in round $r$ and thus received at least $\left\lceil\frac{n+1}{3}\right\rceil$ identical values to $p$. Thus, all processes eventually execute line 25 or 29 in round $r$ and set their own estimate to $v$. After round $r$, the estimate of all processes is 
thus equal to $v$.

Termination The sketch of termination is similar to the case of the ChandraToueg based indirect consensus algorithm. There is a time $t$ such that all faulty processes have crashed. After this time $t$, all correct processes have an estimate $v$ such that $r c v(v)$ holds for at least one correct process. From Hypothesis A, there is thus a time $t^{\prime}$ such that $r c v(v)$ holds for all correct processes and for the estimate $v$ of any correct process. After this time $t^{\prime}$, the indirect consensus algorithm behaves exactly like the original consensus algorithm (albeit with the different level of resilience). Thus, if a decision hasn't been taken before $t^{\prime}$, the Termination property of Mostéfaoui-Raynal's $\diamond \mathcal{S}$ consensus algorithm guarantees that the indirect consensus algorithm also terminates.

No loss In the modified Mostéfaoui-Raynal indirect consensus algorithm, a system is in a $v$-valent configuration if $\left\lceil\frac{2 n+1}{3}\right\rceil$ processes accept the same estimate $v$ in a given round $r$. The estimate of a process is equal to $v$ in three cases : (1) consensus was executed with $v$ as the initial proposal, (2) the process received $v$ in Phase 1 or 2 and accepted it because $m s g s(v)$ had been previously received or (3) the process received $v$ in Phase 2 from at least $f+1$ processes. Since at least $\left\lceil\frac{2 n+1}{3}\right\rceil$ processes have the same estimate $v$ in the $v$-valent configuration, at least $f+1$ processes must have modified their estimate following cases (1) or (2) above. In both of these cases, the processes have $m s g s(v)$. The configuration is thus $v$-stable, since at least $f+1$ processes have $m s g s(v)$. Since any $v$-valent configuration is also $v$-stable, the No loss property is verified.

\section{Performance measurements}

In Section 2, we presented a short performance comparison between atomic broadcast with consensus on messages and consensus on message identifiers. In the following paragraphs, we present measurements comparing atomic broadcast using reliable broadcast and indirect consensus to (the faulty implementation of) atomic broadcast using reliable broadcast and consensus directly on message identifiers, in order to estimate the overhead introduced by the indirect consensus solution.

We also present a comparison between atomic broadcast using indirect consensus to (the correct implementation of) atomic broadcast using uniform reliable broadcast and consensus directly on message identifiers.

This section starts by a presentation of the system setup and the Neko framework that was used in the experiments. Several comparisons between indirect consensus and the faulty and correct implementations using consensus are then 


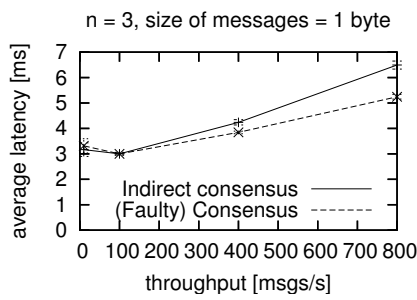

(a) $n=3$ processes

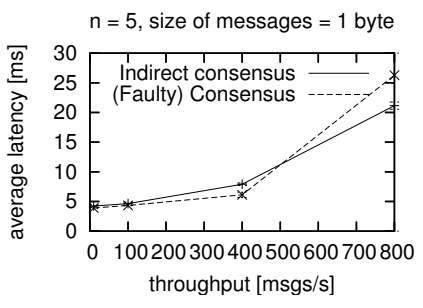

(b) $n=5$ processes

Figure 3: Latency vs. throughput of the atomic broadcast algorithm using indirect consensus or (faulty) consensus on message identifiers in a system with 3 and 5 processes on Setup 1

presented.

\subsection{System setup and the Neko framework}

The benchmarks were run on two setups:

Setup 1 is a cluster of PCs running Red Hat Linux (kernel 2.4.18). The PCs have Pentium III $766 \mathrm{MHz}$ processors and $128 \mathrm{MB}$ of RAM, and are interconnected by a 100 Base-TX Ethernet. The Java Virtual Machine was Sun's JDK 1.4.1_01.

Setup 2 is a cluster of machines running SuSE Linux (kernel 2.6.11). The machines have Pentium 4 processors at $3.2 \mathrm{GHz}$ and have $1 \mathrm{~GB}$ of RAM. They are interconnected by Gigabit Ethernet and run Sun's 1.5.0 Java Virtual Machine.

Neko [9] is a simulation and prototyping framework. Using this framework, the same (Java) implementation of a protocol can be used in a simulated environment and on a real network. The protocols are implemented as layers of a stack. The CT atomic broadcast algorithm was implemented and executed either on messages or on message identifiers, according to the test configuration. The indirect consensus algorithm was implemented based on an already existing implementation of the CT $\checkmark \mathcal{S}$ consensus algorithm that was used in previous performance studies [11, 10]. All the results presented here were obtained on the real networks described above.

\subsection{Performance metric: latency versus throughput and message size}

The performance metric for atomic broadcast is the latency, defined as the average (over all processes) of the elapsed time between abroadcasting a message $m$ and adelivering $\mathrm{m}$. A simple symmetric workload is used: all processes abroadcast messages at the same rate and the global rate is called the throughput.

To quantify the performance of indirect consensus compared to the faulty and 


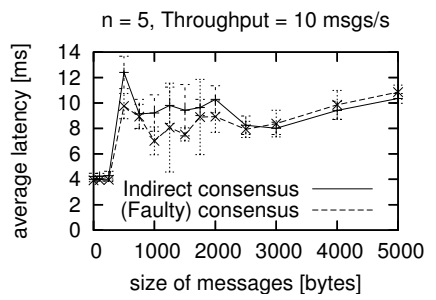

(a) $10 \mathrm{msgs} / \mathrm{s}$

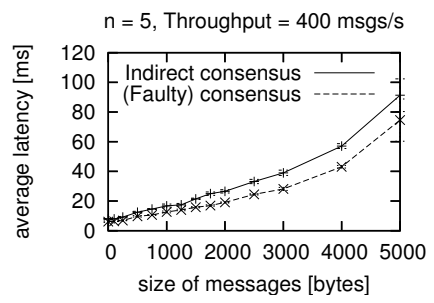

(c) $400 \mathrm{msgs} / \mathrm{s}$

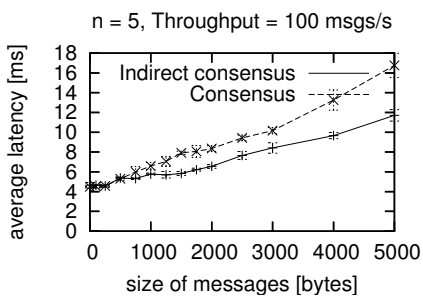

(b) $100 \mathrm{msgs} / \mathrm{s}$

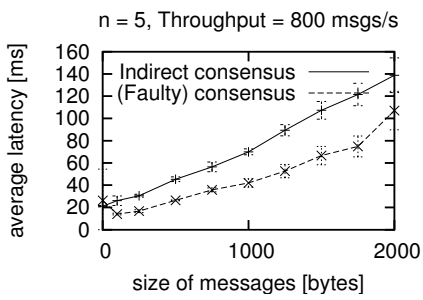

(d) $800 \mathrm{msgs} / \mathrm{s}$

Figure 4: Latency vs. payload of the atomic broadcast algorithm using indirect consensus or (faulty) consensus on message identifiers in a system with 5 processes on Setup 1.

correct implementations of atomic broadcast using consensus directly on message identifiers, we present figures showing the latency of atomic broadcast as a function of the message size (for low and high throughputs) and as a function of the throughput (for a given message size).

\subsection{Performance results: overhead of indirect consensus}

Overhead as a function of the throughput: Figure 3 presents the performance comparison between indirect consensus and the faulty implementation of consensus directly on message identifiers. The size of the messages is set to one byte. Figure 3 shows that the overhead of indirect consensus increases as the throughput increases in a system with 3 or 5 processes in Setup $1 .^{1}$

\footnotetext{
${ }^{1}$ The number of processes might seem small, but is adequate to implement scalable atomic broadcast algorithms. Indeed, in a system with a large amount of processes, there is typically a small kernel of "servers" that order the messages and then broadcast them to all other processes. Thus, only the processes in the kernel actually execute the ordering algorithm. For the sake of efficiency, the set of processes included in the kernel should be small. It is therefore reasonable to compare the performance of consensus algorithms in such a setting.
} 


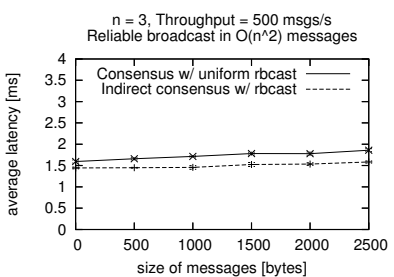

(a) $500 \mathrm{msgs} / \mathrm{s}$

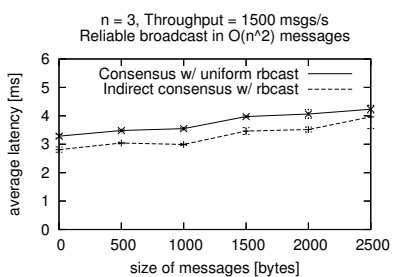

(b) $1500 \mathrm{msgs} / \mathrm{s}$

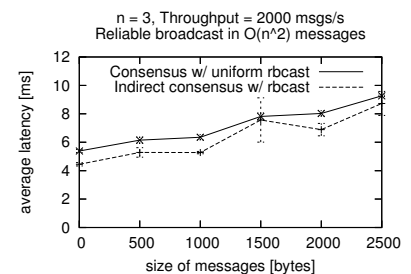

(c) $2000 \mathrm{msgs} / \mathrm{s}$

Figure 5: Latency vs. payload of the atomic broadcast algorithm using indirect consensus and reliable broadcast or consensus on message identifiers and uniform reliable broadcast in a system with 3 processes on Setup 2. The reliable broadcast algorithm uses $O\left(n^{2}\right)$ messages for each rbroadcast.

This result is not surprising: as the throughput increases, consensus is done on larger sets of messages. The calls to the $r c v$ function (to verify if the messages whose identifiers are in a consensus proposal have been received) thus take more and more time. When the throughput is low (10 or 100 messages per second), the overhead of indirect consensus is negligible. As the system throughput increases, the overhead also increases and reaches at most $1.3 \mathrm{~ms}$ in a system with 3 processes and $9.5 \mathrm{~ms}$ in a system with 5 processes. This is the price to pay for a correct implementation, since executing an unmodified consensus algorithm directly on message identifiers can lead to a violation of atomic broadcast's validity property in a system where one process fails.

Overhead as a function of the payload: Figure 4 compares the performance of indirect consensus and consensus directly on message identifiers as the size of the messages increases (in Setup 1). The overhead ratio remains stable as the size of the messages varies. In the case of low throughputs (10 messages per second), the overhead is negligible for all message sizes. For higher throughputs, the overhead is clearly measurable, but does not vary much as the size of the messages increases. These results are expected: since both algorithms only use the message identifiers to reach a decision, the messages themselves (and thus their size) do not affect the performance of the indirect consensus and consensus algorithms.

\subsection{Performance results: comparison of two correct approaches}

Overhead as a function of the payload: Figure 5 and 6 presents the latency of atomic broadcast as a function of the payload of the messages in a system with 3 processes in Setup 2. Atomic broadcast uses either (i) reliable broadcast and 


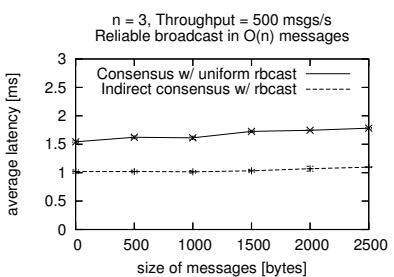

(a) $500 \mathrm{msgs} / \mathrm{s}$

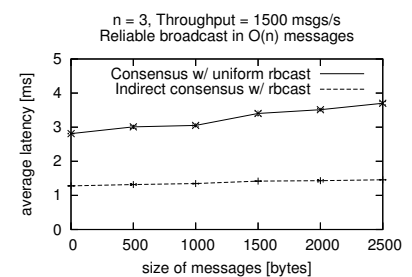

(b) $1500 \mathrm{msgs} / \mathrm{s}$

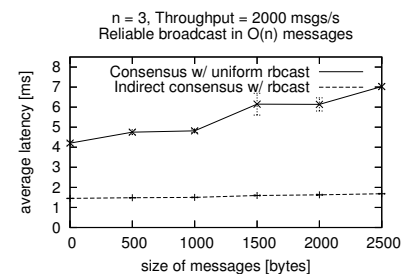

(c) $2000 \mathrm{msgs} / \mathrm{s}$

Figure 6: Latency vs. payload of the atomic broadcast algorithm using indirect consensus and reliable broadcast or consensus on message identifiers and uniform reliable broadcast in a system with 3 processes on Setup 2. The reliable broadcast algorithm uses $O(n)$ messages for each rbroadcast.

indirect consensus or (ii) uniform reliable consensus and consensus directly on message identifiers. Both solutions are correct. The uniform reliable broadcast algorithm that we consider supports up to $f<\frac{n}{2}$ crash-failures and requires $O\left(n^{2}\right)$ messages and 2 communication steps to deliver a message that was previously broadcast.

Figure 5 shows that if reliable broadcast needs $O\left(n^{2}\right)$ messages (as in [2]), then indirect consensus and reliable broadcast achieve slightly lower latencies than consensus on message identifiers and uniform reliable broadcast. This slight difference is attributed to the additional communication step (and message processing) that uniform reliable broadcast needs, compared to reliable broadcast.

Figure 6 shows that if reliable broadcast only needs $O(n)$ messages in good runs (when using a failure detector for example), the performance of indirect consensus is clearly better than if consensus and uniform reliable broadcast are used to solve atomic broadcast.

Overhead as a function of the throughput: Figure 7 presents the latency of atomic broadcast (using either indirect consensus and reliable broadcast or consensus and uniform reliable broadcast) as a function of the throughput in Setup 2. The payload of all messages is one byte. The figure shows that the performance of atomic broadcast using consensus and uniform reliable broadcast degrades significantly as the throughput increases. Atomic broadcast using indirect consensus and reliable broadcast behaves similarly (although slightly better) if reliable broadcast needs $O\left(n^{2}\right)$ messages. If a reliable broadcast algorithm requiring $O(n)$ messages is used however, the performance of atomic broadcast using indirect consensus is much less affected by the throughput than before. 


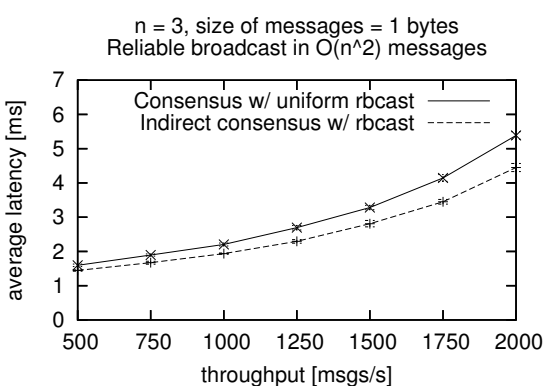

(a) Reliable broadcast in $O\left(n^{2}\right)$

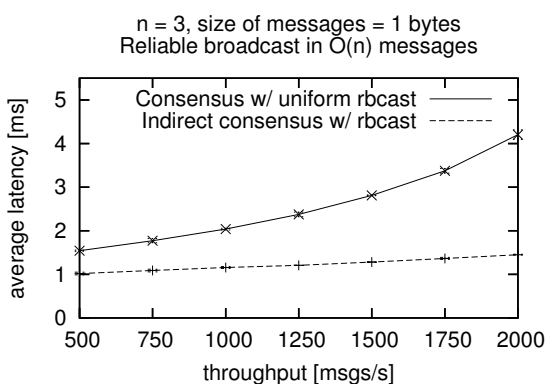

(b) Reliable broadcast in $O(n)$

Figure 7: Latency vs. throughput of the atomic broadcast algorithm using indirect consensus and reliable broadcast or consensus on message identifiers and uniform reliable broadcast in a system with 3 processes on Setup 2. The reliable broadcast algorithm uses either $O\left(n^{2}\right)$ or $O(n)$ messages for each rbroadcast. The payload of each message is 1 byte.

\subsection{Discussion}

In Section 2, we saw that executing atomic broadcast using indirect consensus (on message identifiers) provides better performance than using consensus on messages, especially as the sizes of the system or the messages increase. In previous group communication stack implementations, consensus was often executed directly on message identifiers, which can lead to faulty executions if a process crashes. The indirect consensus approach, which solves this problem, yields performance results that are comparable to the faulty solution (in the case of an indirect consensus algorithm with the same degree of resilience as the corresponding consensus algorithm), as Figures 4 and 3 show.

Furthermore, compared to the other correct solution (atomic broadcast using uniform reliable broadcast and consensus directly on message identifiers), the combination of atomic broadcast and indirect consensus achieves better performance (especially if one considers a reliable broadcast algorithm that only needs $O(n)$ messages per rbroadcast), as illustrated by Figures 5, 6 and 7 .

The cost of adopting a correct implementation of atomic broadcast on message identifiers is thus fairly low and ensures that the properties of atomic broadcast hold, even if processes crash. 


\section{Conclusion}

In [2], atomic broadcast is reduced to consensus on messages. This reduction is correct, but since consensus is executed on sets of messages, it yields poor performance as the size of the messages increases. Instead, consensus can be executed on message identifiers, which decouples the consensus algorithm from the size of the messages. This can however lead to the violation of the Validity property of atomic broadcast. Indirect consensus addresses this issue by providing a No loss property, which guarantees that all messages whose identifiers have been decided upon are eventually delivered by atomic broadcast. To ensure the No loss property, the indirect consensus algorithm must guarantee that any $v$-valent configuration (any future decision is $v$ ) is also $v$-stable (at least one correct process has received the messages whose identifiers are in $v$ ).

The paper has shown that adapting a consensus algorithm into an indirect consensus algorithm is not trivial. The resilience of the adapted Mostéfaoui-Raynal $\checkmark \mathcal{S}$-based indirect consensus algorithm is $f<\frac{n}{3}$ whereas the original consensus algorithm supports $f<\frac{n}{2}$ failures. Chandra-Toueg's $\diamond \mathcal{S}$-based consensus algorithm does not have this problem and was easy to adapt.

Finally, the performance of the Chandra-Toueg based indirect consensus algorithm is better than the original consensus algorithm on messages and comparable to the performance of the faulty implementation of the consensus algorithm directly on message identifiers. The performance of the implementation using indirect consensus algorithm is also better than the performance of atomic broadcast using uniform reliable broadcast and consensus.

\section{References}

[1] M. Castro and B. Liskov. Practical byzantine fault tolerance. In OSDI: Symposium on Operating Systems Design and Implementation. USENIX Association, Co-sponsored by IEEE TCOS and ACM SIGOPS, 1999.

[2] T. D. Chandra and S. Toueg. Unreliable failure detectors for reliable distributed systems. Journal of ACM, 43(2):225-267, 1996.

[3] P. Felber. The CORBA Object Group Service: A Service Approach to Object Groups in CORBA. PhD thesis, École Polytechnique Fédérale de Lausanne, Switzerland, 1998. Number 1867.

[4] M. J. Fischer, N. A. Lynch, and M. S. Paterson. Impossibility of distributed consensus with one faulty process. Journal of ACM, 32(2):374-382, Apr. 1985. 
[5] V. Hadzilacos and S. Toueg. A modular approach to fault-tolerant broadcasts and related problems. TR 94-1425, Dept. of Computer Science, Cornell University, Ithaca, NY, USA, May 1994.

[6] L. Lamport. The part-time parliament. ACM Transactions on Computer Systems, 16(2):133-169, 1998.

[7] A. Mostéfaoui and M. Raynal. Solving consensus using Chandra-Toueg's unreliable failure detectors: A general quorum-based approach. In Proceedings of the 13th International Symposium on Distributed Computing (DISC), number 1693 in Lecture Notes in Computer Science, pages 49-63, Bratislava, Slovak Republic, Sept. 1999. Springer-Verlag.

[8] P. Urbán. Evaluating the Performance of Distributed Agreement Algorithms: Tools, Methodology and Case Studies. PhD thesis, École Polytechnique Fédérale de Lausanne, Switzerland, Aug. 2003. Number 2824.

[9] P. Urbán, X. Défago, and A. Schiper. Neko: A single environment to simulate and prototype distributed algorithms. Journal of Information Science and Engineering, 18(6):981-997, Nov. 2002.

[10] P. Urbán, N. Hayashibara, A. Schiper, and T. Katayama. Performance comparison of a rotating coordinator and a leader based consensus algorithm. In Proc. 23nd IEEE Int'l Symp. on Reliable Distributed Systems (SRDS), pages 4-17, Florianópolis, Brazil, October 2004.

[11] P. Urbán, I. Shnayderman, and A. Schiper. Comparison of failure detectors and group membership: Performance study of two atomic broadcast algorithms. In Proc. of the Int'l Conf. on Dependable Systems and Networks (DSN), pages 645-654, June 2003. 